\title{
Immunological characterization of a gidA mutant strain of Salmonella for potential use in a live-attenuated vaccine
}

\author{
Daniel C Shippy and Amin A Fadl
}

\begin{abstract}
Background: Salmonella is often associated with gastrointestinal disease outbreaks in humans throughout the world due to the consumption of contaminated food. Our previous studies have shown that deletion of glucose-inhibited division gene ( $\mathrm{gidA}$ ) significantly attenuated Salmonella enterica serovar Typhimurium (STM) virulence in both in vitro and in vivo models of infection. Most importantly, immunization with the gidA mutant protected mice from a lethal dose challenge of wild-type STM. In this study, we further characterize the gidA mutant STM strain for potential use in a live-attenuated vaccine.
\end{abstract}

Results: The protective efficacy of immunization with the gidA mutant was evaluated by challenging immunized mice with a lethal dose of wild-type STM. Sera levels of IgG2a and lgG1, passive transfer of sera and cells, and cytokine profiling were performed to study the induction of humoral and cellular immune responses induced by immunization with the gidA mutant strain. Additionally, a lymphocyte proliferation assay was performed to gauge the splenocyte survival in response to treatment with STM cell lysate. Mice immunized with the gidA mutant strain were fully protected from a lethal dose challenge of wild-type STM. Naive mice receiving either cells or sera from immunized mice were partially protected from a lethal dose challenge of wild-type STM. The lymphocyte proliferation assay displayed a significant response of splenocytes from immunized mice when compared to splenocytes from non-immunized control mice. Furthermore, the immunized mice displayed significantly higher levels of $\lg G 1$ and $\lg$ G2a with a marked increase in IgG1. Additionally, immunization with the gidA mutant strain evoked higher levels of IL-2, IFN- $\gamma$, and IL-10 cytokines in splenocytes induced with STM cell lysate.

Conclusions: Together, the results demonstrate that immunization with the gidA mutant strain protects mice by inducing humoral and cellular immune responses with the humoral immune response potentially being the main mechanism of protection.

Keywords: Salmonella, GidA, Vaccine, Immune responses, Mouse model

\section{Background}

Salmonella is an enteric pathogen causing major public health problems throughout the world due to the consumption of contaminated food. Nontyphoidal Salmonella species, like Salmonella enterica serovar Typhimurium (STM), are the leading cause of hospitalization and death among the major foodborne pathogens [1]. Antibiotic resistance by Salmonella is dramatically

\footnotetext{
* Correspondence: fadl@wisc.edu

Department of Animal Sciences, University of Wisconsin-Madison, 1675 Observatory Dr, Madison, WI 53706, USA
}

(c) 2012 Shippy and Fadl; licensee BioMed Central Ltd. This is an Open Access article distributed under the terms of the Creative Commons Attribution License (http://creativecommons.org/licenses/by/2.0), which permits unrestricted use, distribution, and reproduction in any medium, provided the original work is properly cited. remains a global health priority $[2,3]$.

Creating a safe and immunogenic vaccine strain is the biggest challenge in developing an effective liveattenuated Salmonella vaccine [4]. Several Salmonella vaccines, including whole-cell killed and live vaccines, have been developed with variable success $[5,6]$. These vaccines either required repeated administration or induced insufficient immune responses for long-lasting protection against lethal challenges with virulence Salmonella strains [7]. Many Salmonella vaccine strains carry deletion mutations affecting metabolic functions or virulence factors [8]. Several mutant strains of 
Salmonella have been investigated in the pursuit to develop optimal immune responses [9-11]. Our approach in constructing a live-attenuated Salmonella vaccine strain is to create a mutant defective in tRNA modification [12]. This strategy enables our vaccine strain to express multiple virulence factors at a significantly reduced level in order to obtain a safe and immunogenic vaccine candidate.

Glucose-inhibited division (GidA) protein (also known as MnmG) was first described in Escherichia coli, where deletion of gidA resulted in a filamentous morphology when grown in a rich medium supplemented with glucose [13]. Further studies showed GidA is a flavin dinucleotide (FAD) binding enzyme involved in the fruiting body development of Myxococcus xanthus [14]. Furthermore, GidA has been shown to be a tRNA modification methylase in $E$. coli that forms a heterodimeric complex with MnmE (also known as TrmE) to catalyze the addition of a carboxymethylaminomethyl $(\mathrm{cmnm})$ group at the 5 position of the wobble uridine (U34) of tRNAs [15-19]. Most importantly, deletion of gidA has been shown to attenuate the pathogenesis of some bacteria including Pseudomonas syringae, Aeromonas hydrophila, Streptococcus pyogenes, and Pseudomonas aeruginosa [20-23].

Our previous studies suggest a role for GidA in the regulation of Salmonella virulence and cell division $[12,24]$. In our initial study, the gidA mutant was attenuated in vitro and showed a significant decrease in ability to invade T84 intestinal epithelial cells as well as a significant decrease in ability to replicate and produce cytotoxic affects on macrophages. Furthermore, global transcriptional and proteomic profiling indicated a significant down-regulation in numerous genes and proteins involved in Salmonella pathogenesis [12]. Most importantly, the gidA mutant was attenuated in mice as shown by a significant increase in 50\% lethal dose $\left(\mathrm{LD}_{50}\right)$, reduced systemic bacterial survival, defective in the induction of inflammatory cytokines and chemokines, and reduced severity of histopathological lesions in the liver and spleen. Additionally, mice immunized with the gidA mutant were protected from a lethal dose challenge of wild-type (WT) STM [12].

In this study, we examined the relative contribution of the humoral and cellular immune responses in the overall protective mechanism afforded by immunization with the gidA mutant STM strain to further evaluate it as a candidate for use in a live-attenuated vaccine. The protective efficacy of immunization with the gidA mutant was evaluated by challenging immunized mice with a lethal dose of WT STM. Sera of control and immunized mice were tested for levels of IgG1 and IgG2a to gauge the Th1 and Th2 responses to gidA immunization. Additionally, sera and cell culture supernatant were used to determine the level of induction of Th1 (IL-2 and IFN- $\gamma$ ) and Th2 (IL-4 and IL-10) cytokines in control and immunized mice. Passive transfer studies were performed to evaluate the role of humoral and cell mediated immunity afforded by immunization with the gidA mutant vaccine strain. A lymphocyte proliferation assay was used to determine the ability of control and immunized murine splenocytes to respond to treatment with STM cell lysate. Taken together, these data indicate the gidA mutant vaccine strain protects mice by inducing humoral and cellular immune responses with the humoral immune response being the primary mechanism of protection.

\section{Methods}

\section{Bacterial strains and growth conditions}

The WT and gidA mutant Salmonella enterica serovar Typhimurium (STM) 14028 strains are described in [12]. The organisms were grown in Luria-Bertani (LB) broth and on LB agar plates in the presence of nalidixic acid $(150 \mu \mathrm{g} / \mathrm{ml})$ or kanamycin $(50 \mu \mathrm{g} / \mathrm{ml})$. The bacteria were cultivated at $37^{\circ} \mathrm{C}$ with shaking at $225 \mathrm{rpm}$. Bacteria were harvested by centrifugation (5,000 rpm for $10 \mathrm{~min}$ ), washed twice with PBS, and resuspended in a minimal amount of PBS.

\section{Immunization of mice}

Female BALB/c mice, 6-8 weeks old, were obtained from Harlan Laboratories (Indianapolis, IN). All animal procedures were approved by the University of Wisconsin-Madison Animal Care and Use Committee. Mice were kept under specific pathogen-free conditions in filter-topped cages and provided with food and water ad libitum. Mice were inoculated via the intraperitoneal (i.p.) route with either $1 \times 10^{3} \mathrm{CFU}$ of the gidA mutant STM strain, or sterile PBS. The chosen time points for the assays in this study are 7 and 42 days after immunization. These time points were chosen to gauge the immune response to the gidA mutant STM strain at the early stage of infection and at the time of challenge. At these time points, mice were sedated with isoflurane (Abbott Laboratories, North Chicago, IL) and bled for sera which were used to profile the Th1 and Th2 cytokines, determine the IgG subclasses, and used in the passive transfer experiment. The spleens were removed and these cells were used for the cell population analysis, lymphocyte proliferation assay, Th1 and Th2 cytokine profiling, and the passive transfer experiment. At the 42 day time-point, selected mice that had been injected with PBS and the gidA mutant STM strain were challenged with a lethal dose $\left(1 \times 10^{5} \mathrm{CFU}\right)$ of WT STM. Morbidity and mortality of these animals were monitored for 30 days after challenge. Mice suffering from lethal salmonellosis as determined by severe hunched 
posture, labored breathing, apathy, and ruffled fur were euthanized to prevent unnecessary suffering.

\section{Splenic bacterial counts}

The enumeration of bacteria from the spleen was performed as previously described [12]. Briefly, spleen samples of $0.1 \mathrm{~g}$ were removed from mice inoculated with sterile PBS or the gidA mutant STM strain, homogenized in $1 \mathrm{ml}$ PBS, and serial dilutions of the homogenate were plated on Salmonella-Shigella (SS) and LB agar plates. The plates were incubated at $37^{\circ} \mathrm{C}$ for 24 hours and colonies were counted. Bacteria were enumerated by determining the CFU in duplicate, and expressed as $\mathrm{CFU} / \mathrm{ml}$.

\section{Flow cytometric analysis}

Spleens were removed from mice inoculated with sterile PBS or the gidA mutant STM strain. The spleens were homogenized in RPMI media supplemented with $2 \%$ fetal bovine serum (FBS), filtered through a $70 \mu \mathrm{m}$ strainer, and the red blood cells were lysed with Pharm Lyse cell lysis buffer (BD Bioscience, Franklin Lakes, NJ). The spleen cells were washed twice with PBS supplemented with $2 \%$ FBS, filtered through a $70 \mu \mathrm{m}$ strainer, and counted on a hemocytometer. Approximately $1 \times 10^{6}$ cells were placed in each tube, and incubated with mouse CD16/CD32 monoclonal antibodies $(0.25 \mu \mathrm{g} / 100 \mu \mathrm{l})$ (BD Bioscience) for $15 \mathrm{~min}$ at room temperature to block antibody binding to mouse $\mathrm{Fc}-\gamma$ receptors. The cells were washed twice with PBS supplemented with 2\% FBS and incubated with either antiCD4 antibody conjugated to PE-Cy5 $(0.20 \mu \mathrm{g} / 100 \mu \mathrm{l})$ or anti-CD8 antibody conjugated to PE-Cy7 $(0.30 \mu \mathrm{g} / 100 \mu \mathrm{l})$ and anti-CD44 antibody conjugated to fluorescein isothiocyanate (FITC) $(0.20 \mu \mathrm{g} / 100 \mu \mathrm{l})$ and anti-CD62L antibody conjugated to phycoerythrin (PE) $(0.10 \mu \mathrm{g} /$ $100 \mu \mathrm{l})$. After incubation, the cells were washed once with PBS supplemented with $2 \%$ FBS and fixed with $1 \%$ formaldehyde. Analysis was performed at the University of Wisconsin-Madison Carbone Cancer Center Flow Cytometry Laboratory using a LSRII flow cytometer and FlowJo software (Tree Star Inc., Ashland, OR).

\section{ELISA}

Initially, a whole-cell Salmonella enzyme-linked immunosorbent assay (ELISA) was performed as previously described [25]. The purpose of this experiment is to assay the serum antibody specific for our gidA mutant STM strain. Serum IgG1 and IgG2a from mice inoculated with sterile PBS or the gidA mutant STM strain was measured 7 and 42 days post-immunization by ELISA as previously described [10]. High-binding flatbottom ELISA plates (Thermo Fisher Scientific, Rochester, NY) were coated with $1 \mu \mathrm{g} / \mathrm{ml}$ of capture antibody
(anti-IgG1 or anti-IgG2a) (Bethyl Laboratories Inc., Montgomery, TX) diluted in $0.05 \mathrm{M}$ carbonate/bicarbonate buffer ( $\mathrm{pH}$ 9.6) for 1 hour at room temperature. The wells of the microtiter plate were washed five times with washing buffer $(50 \mathrm{mM}$ Tris, $0.14 \mathrm{M} \mathrm{NaCl}$, and $0.05 \%$ Tween 20) and blocked with blocking buffer $(50 \mathrm{mM}$ Tris, $0.14 \mathrm{M} \mathrm{NaCl}$, and $1 \%$ bovine serum albumin [BSA]) overnight at $4^{\circ} \mathrm{C}$. After washing, sera from both groups of mice were diluted in sample buffer $(50 \mathrm{mM}$ Tris, $0.14 \mathrm{M} \mathrm{NaCl}, 1 \% \mathrm{BSA}$, and $0.05 \%$ Tween 20) and the Mouse Reference Serum (Bethyl Laboratories Inc.) was diluted two-fold starting at a concentration of $1000 \mathrm{ng} / \mathrm{ml}$ and used for plotting the standard curve. After one hour incubation at room temperature, the plates were washed five times with washing buffer, and incubated for an additional hour at room temperature after the addition of a 1:250,000 dilution of horseradish peroxidase (HRP)-conjugated goat anti-mouse IgG (Bethyl Inc.) to the wells of the microtiter plate. After washing five times, 3, 3, 5, 5' tetramethylbenzidine (TMB) substrate was added to visualize antigen-antibody reactions. The reaction was stopped with $0.18 \mathrm{M} \mathrm{H}_{2} \mathrm{SO}_{4}$, and the optical density was measured at $450 \mathrm{~nm}$.

\section{Lymphocyte proliferation assay}

The lymphocyte proliferation assay was performed using the described method [26]. Splenocytes harvested on day 7 and 42 post-immunization were used in the lymphocyte proliferation assay. After harvesting, live splenocytes were determined by the trypan blue exclusion technique and counting with a hemocytometer. Cells from both groups of mice were plated in a 96-well U-bottom microtiter plate (Corning Inc., Corning, NY) at a cell density of $2 \times 10^{5}$ cells/well. The cells were treated with STM cell lysate $(1 \mu \mathrm{g} / \mathrm{ml})$ and incubated at $37^{\circ} \mathrm{C}$ with $5 \%$ $\mathrm{CO}_{2}$ for 48 hours. The STM cell lysate was created from a WT STM 14028 culture that was grown to an optical density (O.D.) $)_{600}$ of 1.0, washed twice with PBS, lysed by sonication, and quantitated using a Bradford Assay. The percentage of cell survival was determined using the CytoTox-Glo Cytotoxicity Assay (Promega, Madison, WI). Quantification of viable cells was determined by the formula: Signal from Viable Cells = Total Cytotoxicity Signal - Initial Cytotoxicity Signal.

\section{Cytokine profiling}

The cytokine profiling was performed using a commercially based multiplex assay as described [12]. Th1 (IL-2 and IFN- $\gamma$ ) and Th2 (IL-4 and IL-10) cytokine levels were determined from mouse sera at day 7 and 42 using a multiplex assay (Quansys Biosciences, Logan, UT). Cytokine production from splenocytes at day 7 and 42 was measured by plating splenocytes from both groups of mice in a microtiter plate at a cell density of $2 \times 10^{5}$ 
cells/well. The cells were treated with STM cell lysate $(1 \mu \mathrm{g} / \mathrm{ml})$ and incubated at $37^{\circ} \mathrm{C}$ with $5 \% \mathrm{CO}_{2}$ for 48 hours. The levels of Th1 and Th2 cytokines in the culture supernatant were determined using a multiplex assay (Quansys Biosciences).

\section{Passive transfer of cells and sera}

Mice were bled for sera and splenocytes were harvested on day 42 post-immunization. Fifteen naïve mice were used with the mice being divided into three groups with five mice per group. Each group was inoculated via retro-orbital injection [27] with either $100 \mu \mathrm{l}$ sterile PBS, $100 \mu \mathrm{l}$ of sera from non-infected mice, or $100 \mu \mathrm{l}$ of sera from mice immunized with the gidA mutant STM strain [28]. Another fifteen naïve mice were divided into three groups of five and each group was inoculated via retroorbital injection [27] with $100 \mu \mathrm{l}$ sterile PBS, $100 \mu \mathrm{l}$ of splenocytes $\left(1 \mathrm{x} \quad 10^{7}\right.$ cells $\left./ 100 \mu \mathrm{l}\right)$ from non-infected mice, or $100 \mu \mathrm{l}$ of splenocytes $\left(1 \times 10^{7}\right.$ cells $\left./ 100 \mu \mathrm{l}\right)$ from mice immunized with the gidA mutant STM strain [28]. All groups were challenged by i.p. injection 24 hours later with a lethal dose $\left(1 \times 10^{5} \mathrm{CFU}\right)$ of WT STM. Morbidity and mortality of these animals were monitored for 30 days after challenge. Mice suffering from lethal salmonellosis as determined by severe hunched posture, labored breathing, apathy, and ruffled fur were euthanized to prevent unnecessary suffering.

\section{Statistical analysis}

Wherever appropriate, the data were analyzed using GraphPad Prism 5 software (GraphPad Software, San Diego, CA) and a Student's $t$ test. $P$ values of $\leq 0.05$ were considered significant, and data were expressed as arithmetic means with standard deviations. Animal mortality was analyzed using the Kaplan-Meier survival analysis with the log-rank (Mantel-Cox) significance test.

\section{Results}

Protective efficacy of the gidA mutant STM strain

To examine the protection provided by GidA immunization, six BALB/c mice were i.p. injected with sterile PBS while another six mice were injected with $1 \times 10^{3}$ CFU of the gidA mutant STM strain. AT 42 days postimmunization, all twelve mice were challenged with a lethal dose $\left(1 \times 10^{5} \mathrm{CFU}\right)$ of WT STM. All of the control mice challenged with the WT STM strain died within four days of challenge. Meanwhile, all of the mice immunized with the gidA mutant STM strain survived the lethal dose challenge of WT STM. Furthermore, none of the mice immunized with the gidA mutant STM strain showed any lack of mobility, hunched posture, or ruffled fur associated with septic shock (Figure 1).

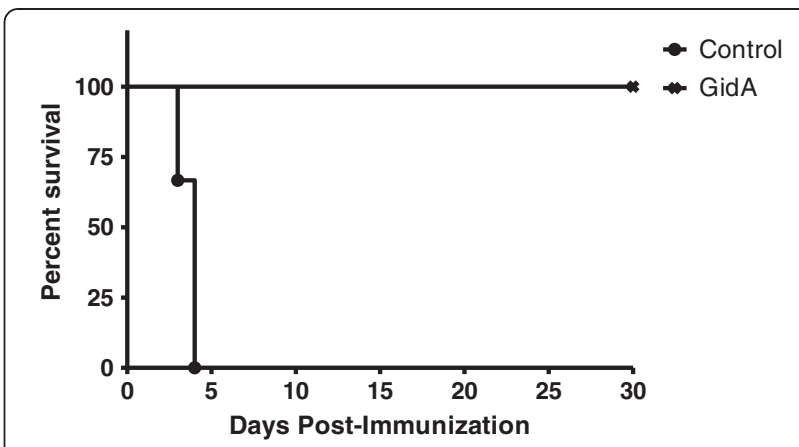

Figure 1 Percent survival of mice immunized by i.p. injection with sterile PBS or $1 \times 10^{3}$ CFU of the gidA mutant vaccine strain, and subsequently challenged with a lethal dose $\left(1 \times 10^{5}\right.$ (FU) of WT STM on day 42 post-immunization. Morbidity and mortality of these animals were monitored for 30 days after challenge. Full protection was provided to immunized mice while $100 \%$ mortality was seen in the control mice.

\section{Splenic bacterial counts after immunization}

We previously reported the level of bacteria recovered from spleens of mice inoculated with the gidA mutant STM strain was significantly less than that recovered from spleens of mice inoculated with the WT STM strain [12]. In this study, the in vivo stability of the gidA mutant STM strain was determined by examining its ability to colonize the spleen at Day 7 and at the time of challenge (Day 42). The number of viable bacteria recovered from mice immunized with the gidA mutant STM strain was $4.0 \operatorname{logs}$ on day 7 post-immunization. At day 42 post-immunization, viable bacteria were still recovered from the spleen at 0.9 logs (Figure 2). The long persistence of the bacteria in mouse splenic tissues could

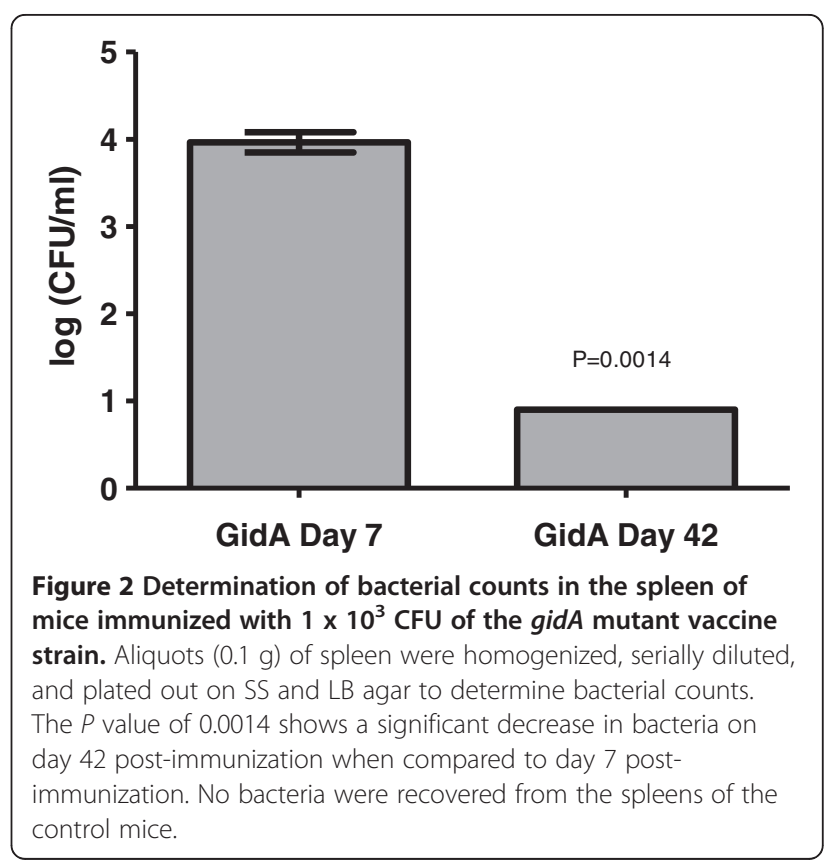


enable sustained immune response activities in mice immunized with the gidA mutant STM strain. As shown by the histopathological grading in our initial GidA study, the colonization of the gidA mutant STM strain only caused mild necrosis with moderate infiltration of inflammatory cells in the spleen with little to no induction of proinflammatory cytokines and chemokines [12].

\section{T cell analysis in mice immunized with the gidA mutant STM strain}

To determine whether $\mathrm{T}$ cells were activated in BALB/C mice immunized with $1 \times 10^{3} \mathrm{CFU}$ of the gidA mutant STM strain, isolated splenocytes from control and immunized mice were harvested at day 7 and 42 postimmunization. Splenocytes from both groups of mice were stained with antibodies against CD4 or CD8 in combination with anti-CD44 and anti-CD62L antibodies. These markers are used to distinguish naïve from activated or memory $\mathrm{T}$ cells [29]. The level of $\mathrm{CD}^{+}$cells were higher in the immunized mice $(21.3 \%)$ when compared to the control mice $(16.1 \%)$ on day 7 and again on day 42 (28.1 and $23.5 \%$, respectively). There was no difference in the $\mathrm{CD}^{+}$populations between the control and immunized mice on day 7 and 42 . Furthermore, on day 7 and 42 post-immunization, there was no significant difference between the control and gidA mutant immunized mice in the percentage of $\mathrm{CD} 44^{+}$and $\mathrm{CD} 2 \mathrm{~L}^{+}$in both $\mathrm{CD} 4^{+}$and $\mathrm{CD} 8^{+} \mathrm{T}$ cells (data not shown).

\section{Serum IgG levels in mice after immunization}

The Salmonella whole cell ELISA displayed a high-level of Salmonella specific antibody. In order to further characterize the immune response elicited after immunization with the gidA mutant STM strain, the sera of control and immunized mice were examined for the production of IgG2a and IgG1 antibodies as markers of Th1 and Th2 subsets, respectively. These findings indicate a significant increase in both IgG2a $[P=0.0317$ and $P=0.0179$ for GidA day 7 and 42 , respectively, compared to the control] and IgG1 $[P=0.0051$ and $P=0.0007]$ in the sera of mice immunized with the gidA mutant STM strain with the highest levels being assayed on day 42 post-immunization. Furthermore, the IgG1 response, indicative of Th2, was higher in the immunized mice than the IgG2a response level in the immunized mice (Figure 3).

\section{Lymphocyte proliferation assay}

Splenocytes harvested from control mice and mice immunized with the gidA mutant strain were used to examine the cellular immune response against treatment with STM cell lysate. At day 7 post-immunization, the splenocytes from the immunized mice displayed a
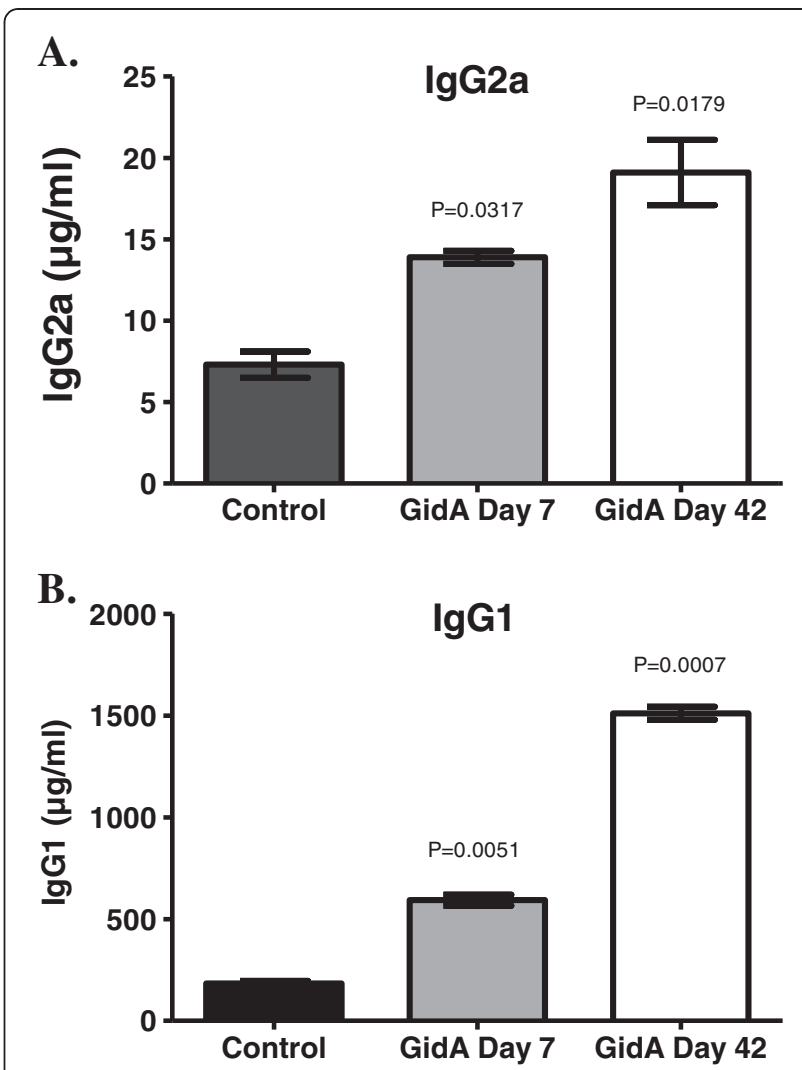

Figure $3 \mathrm{BALB} / \mathrm{c}$ mice were immunized with $1 \times 10^{3} \mathrm{CFU}$ of the gidA mutant vaccine strain or sterile PBS. Serum $\operatorname{lgG} 2 \mathrm{a}(\mathbf{A})$ and serum IgG1 (B) concentrations were determined by ELISA at the indicated times after immunization. The actual $P$ values are provided comparing the sera levels of the immunized mice to that of the control group.

significant proliferative response $(932,590) \quad[P=0.0113]$ compared to the splenocytes from the control mice $(365,910)$. Once again, at 42 days post-immunization, the splenocytes from the immunized mice showed a significantly higher proliferative response $(411,177)$ $[P=0.0282]$ than the splenocytes from control mice $(81,574)$ when treated with STM cell lysate. In contrast, splenocytes from non-immunized control mice showed little proliferation in response to treatment with the STM cell lysate (Figure 4).

\section{Cytokine analysis}

Sera and splenocyte cell culture supernatants were examined for both Th1 (IL-2 and IFN- $\gamma$ ) and Th2 cytokines (IL-4 and IL-10). The sera of mice immunized with the gidA mutant STM strain showed no difference from that of the control sera in the level of cytokine induction on days 7 and 42 post-immunization (data not shown). These data confirm the findings in our initial GidA study which showed a marked reduction in the levels of all of the major cytokines when compared to sera of 


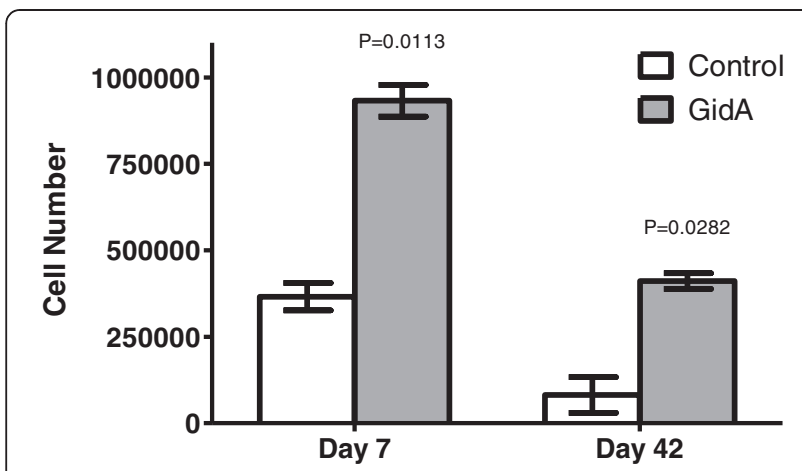

Figure 4 Lymphocyte proliferation assay displaying the survival of splenocytes from control and immunized mice before and after treatment with STM cell lysate. The actual $P$ values for the given time points are provided showing the significant increase in proliferation in splenocytes from immunized mice in comparison to splenocytes from control mice.

mice infected with the WT STM strain [12]. In the cell culture supernatant, the induction of Th1 and Th2 cytokines were significantly increased when GidA splenocytes were induced with STM cell lysate. Meanwhile, there was little to no cytokine induction in the cell culture supernatant when splenocytes from control mice were treated with the STM cell lysate. Furthermore, there was no IL-4 induction in either the control or GidA groups at days 7 and 42 (data not shown). On days 7 and 42 post-immunization, there was no difference between the treated and untreated control groups in the level of IL-2 induction. The level of IL-2 induction, however, significantly increased in the GidA treated cells (Figure 5A) $[P=0.0007$ and $P<0.0001]$. The level of IFN- $\gamma$ displayed a slight increase in the control treated cells $(11.8 \mathrm{pg} / \mathrm{ml})$ over the untreated control cells $(0.3 \mathrm{pg} / \mathrm{ml})$ on day 7 , but showed no difference on day 42. In contrast, the GidA treated cells showed a marked increase in IFN- $\gamma$ induction (1388.4 and $108.2 \mathrm{pg} / \mathrm{ml}$ ) $[P<0.0001$ and $P=0.0001]$ compared to the untreated GidA cells $(0.3$ and $0.3 \mathrm{pg} / \mathrm{ml})$ on days 7 and 42 , respectively (Figure $5 \mathrm{~B}$ ). The levels of IL-10 were similar between the control groups on day 7, but the level of IL-10 induction in the GidA treated cells were significantly higher than that of the GidA untreated cells $[P=0.0001]$. On day 42 , there was no difference in IL-10 induction in either the control or GidA group (Figure $5 \mathrm{C}$ ). Together, these results indicate that immunization with the gidA mutant STM strain elicited a mixed Th1 and Th2 response when treated with STM cell lysate.

\section{Protective efficacy of cells and sera}

A passive-immunization study was performed in order to evaluate the roles of antibody and cell mediated immunity provided by immunization of mice with the gidA mutant STM strain. Spleen lymphocytes $\left(\begin{array}{llll}1 & \mathrm{x} & 10^{7} & \text { cells/ }\end{array}\right.$
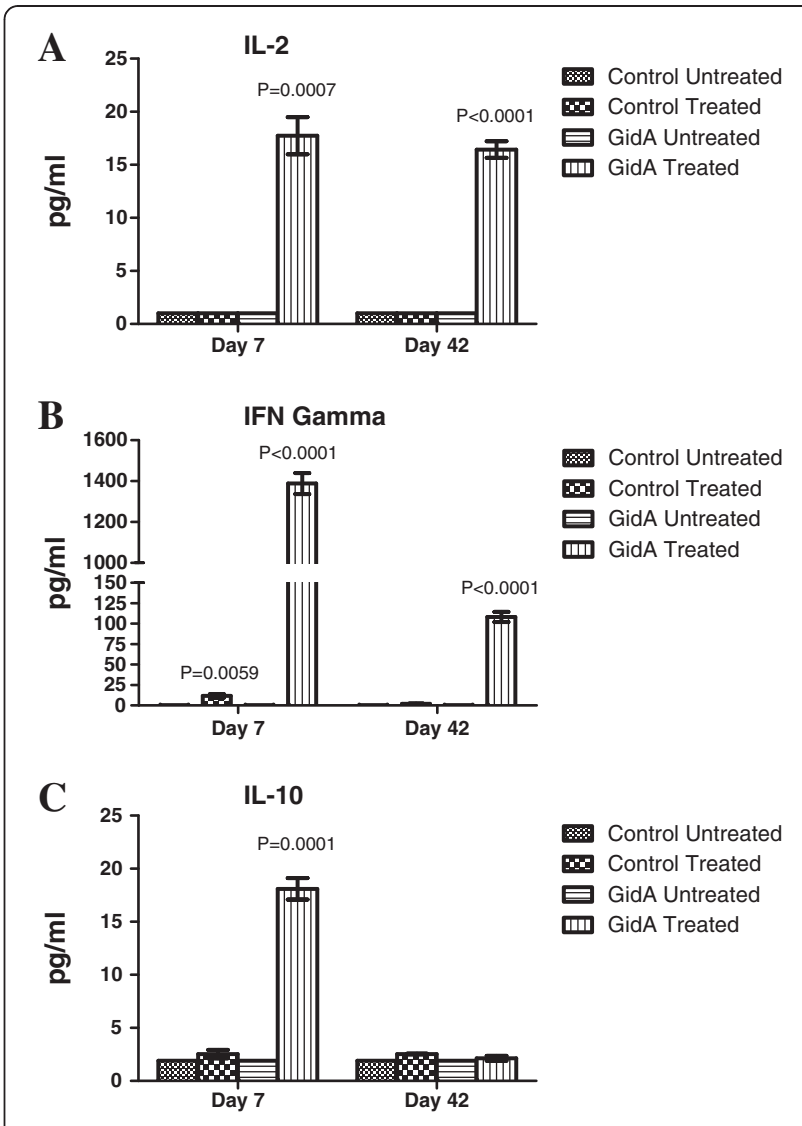

Figure 5 Induction of IL-2, IFN- $\gamma$, and IL-10 in the cell culture supernatant from control and immunized mice before and after treatment with STM cell lysate. Splenocytes were collected from both groups of mice at days 7 and 42 post-immunization and the levels of IL-2 (A), IFN- $\gamma(\mathbf{B})$, and IL-10 (C) was determined using a multiplex assay. The actual $P$ values are given for each time point.

$100 \mu \mathrm{l})$ or $100 \mu \mathrm{l}$ of pooled sera taken from immunized mice or controls was administered by retro-orbital injection into groups of five naïve mice. Another group of five naïve mice was injected with sterile PBS to serve as an additional control. Approximately 24 hours later, all mice were challenged with a lethal dose $\left(1 \times 10^{5} \mathrm{CFU}\right)$ of the WT STM strain. All of the mice receiving control sera, control cells, or sterile PBS died within four days of being challenged by the WT STM strain. The sera transferred from the gidA mutant immunized mice protected three of the five naïve mice from challenge. Furthermore, the two mice in this group that died showed a delay in death (7 and 8 days following challenge) when compared to the control serum and PBS control groups (Figure 6A). The cells transferred from the gidA mutant immunized mice protected two of the five naïve mice from challenge. The three mice that died from this group died in the same time period as mice receiving control cells and PBS (Figure 6B). From these data both parts of the immune response are somewhat protective, 

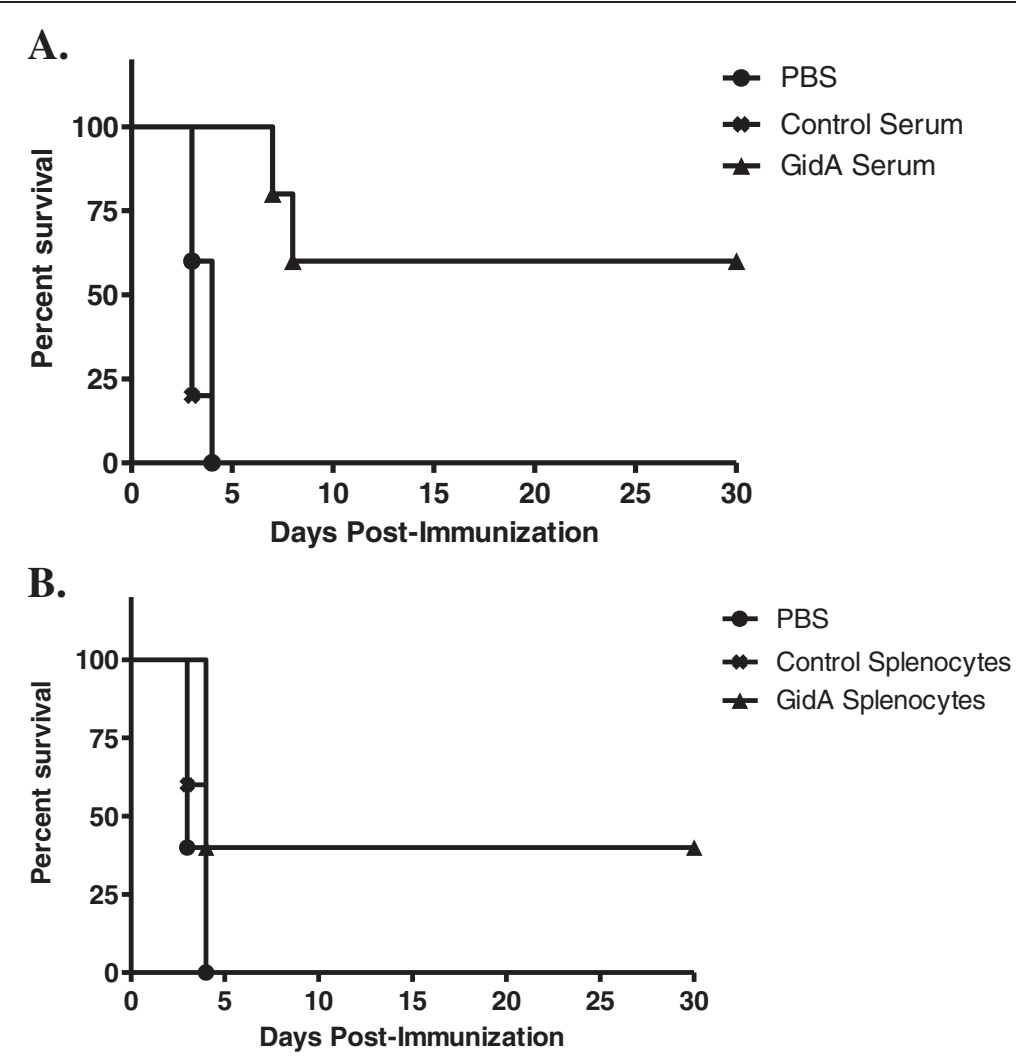

Figure 6 Mice were immunized with $1 \times 10^{3} \mathrm{CFU}$ of the gidA mutant vaccine strain or sterile PBS. Serum and cells were collected 42 days later and transferred to groups of five naïve mice. All recipient mice were challenge by i.p. injection with $1 \times 10^{5}$ CFU of WT STM 24 hours after transfer. Morbidity and mortality of these animals were monitored for 30 days after challenge. The serum passive transfer (A) was statistically significant with a $P$ value of 0.0414 while the cell passive transfer $(\mathbf{B})$ was not statistically significant. Statistical significance was calculated using the Kaplan-Meier survival analysis with the log-rank (Mantel-Cox) significance test.

but antibody mediated immunity appears to be the more crucial of the two in protecting mice from WT STM.

\section{Discussion}

In this study, for the first time, the mechanism of protection provided by immunization with the gidA mutant STM strain was characterized. GidA was originally thought to be involved in cell division due to the filamentous morphology observed when the cells were grown in rich medium supplemented with glucose [13]. More recent studies done in E. coli have shown GidA modulates several bacterial factors by a post-transcriptional mechanism to modify tRNA by the addition of a cmnm group at the 5 position of the wobble uridine (U34) of tRNAs [15-19,30,31]. It has been proposed that tRNA modification can serve as a regulatory mechanism to modulate gene expression[32]. Furthermore, it has been suggested that secreted proteins are particularly vulnerable to U34 hypomodification, and many codons in bacteria require proper U34 modification for efficient decoding [33]. Studies will need to be conducted in Salmonella to see if GidA modifies tRNA in the same fashion as in E. coli. Such studies are currently underway in this laboratory.

Immunization of mice with the gidA STM mutant strain provided full protection from a lethal dose challenge of WT STM. All of the immunized mice survived a lethal dose challenge, while all the naïve mice died within 4 days of challenge. Furthermore, none of the immunized mice displayed any visual signs of illness or septic shock associated with Salmonella infection. We chose to challenge the immunized mice with a WT STM dose of $1 \times 10^{5} \mathrm{CFU}$ which is highly lethal. In our initial GidA study, this dose was approximately 1000 times higher than the $\mathrm{LD}_{50}$ of the WT STM strain [12]. We chose such a high challenge dose because we feel it is more reflective of the amount of Salmonella animals are exposed to in the environment.

Antibody responses are known to contribute to Salmonella immunity [34-36]. It has been proposed that antibodies made by IgM memory B cells are the firstline defense mechanism against all infections and these antibodies are the only defense against $\mathrm{T}$ cellindependent antigens [37]. Studies in B cell deficient 
mice have shown that B cells are required for efficient protection from both primary and secondary Salmonella infection [36]. Our data indicates a strong humoral response to immunization with the gidA mutant STM strain. The Th2 marker, IgG1, showed a marked increase in sera of mice immunized with the gidA mutant STM strain. Naïve mice receiving sera from immunized mice were more protected than naïve mice receiving a passive transfer of cells from immunized mice. Further, the level of the Th2 cytokine IL-10 showed a significant increase in induction when splenocytes from immunized mice were treated with STM cell lysate. The strong Th2 response, however, was not accompanied by an increase in IL-4 induction. IL-4, along with IL-10, induces differentiation of uncommitted $\mathrm{T}$ cells toward a Th2 phenotype $[38,39]$. One possible explanation for this could be reasoned from the study by Okahashi et al. In their study, IL-4 knockout mice which were unable to generate classical Th2-type responses were still capable of producing significant antibody responses to inoculation with Salmonella [40].

Since Salmonella is a facultative intracellular pathogen, cellular immune responses are considered to be a crucial component of protective immunity. Protective cellular mediated immunity is mediated by $\mathrm{CD}^{+}$cells which results in the activation of macrophages and delayedtype hypersensitivity responses. Numerous gene target studies have shown the importance of $\mathrm{CD} 4^{+}$activation in resistance to Salmonella infection [41,42]. Our data indicates a cellular immune response in mice immunized with the gidA mutant STM strain. Although the flow cytometric analysis showed no induction of memory $\mathrm{T}$ cells, or difference in $\mathrm{CD}^{+}$cells, it shows an increase in $\mathrm{CD} 4^{+}$population in the immunized mice at both day 7 and 42 post-immunization. It has been shown that $\mathrm{CD} 4^{+}$ cells are more important than $\mathrm{CD}^{+}$in resistance to Salmonella infection $[43,44]$. The passive transfer of cells to naïve mice from immunized mice did not confer full protection, and was not as significant as the serum passive transfer, but there was enough cell mediated immunity activated to protect a portion of the mice from a lethal dose challenge. Furthermore, splenocytes from immunized mice proliferated at a much higher rate than splenocytes from control mice when treated with STM cell lysate. The IgG1 induction was significantly more prominent than the induction of IgG2a, but the level of IgG2a was still significantly higher in the immunized mice than in that of the sera of the control mice. Furthermore, the induction of the Th1 cytokines, IL-2 and IFN- $\gamma$, shows a strong indication of cell mediated immunity induced by immunization. In particular, IFN- $\gamma$ showed a marked increase in cell culture supernatant when splenocytes from immunized mice were treated with STM cell lysate.
The general consensus is that the ideal Salmonella vaccine should generate both humoral and cell mediated immunity. This is due to protective immunity to Salmonella in mice being attributed to a balance between humoral and cell mediated immunity with an emphasis on development of the Th1 and Th2 subsets $[45,46]$. In this study, the gidA mutant vaccine strain generated both Th1 and Th2 immunity with the Th2 immune response being the more prominent of the two. This was somewhat surprising since Salmonella is a facultative intracellular pathogen. One possible explanation for this could be found in our initial GidA study comparing the gidA mutant to the WT STM strain. The gidA mutant showed an approximate 1000-fold reduction in the ability to invade T84 intestinal epithelial cells, as well as a marked reduction in ability to cause systemic infection in mice. Additionally, transcriptional and proteomic profiling identified a significant down-regulation in numerous genes and proteins responsible for invasion. Overall, the gidA mutant vaccine strain provides full protection to mice when challenged with a highly lethal dose of WT STM. The passive transfer experiments show the importance of both humoral and cell mediated immunity in this protective mechanism. This is an initial study in which a proof of principle of protective immunity has been established suggesting a gidA mutant STM strain could be a good candidate for use in a live-attenuated Salmonella vaccine. Future studies will be conducted using oral immunization in order to establish the optimal immunization route. Once the immunization route is established, further studies will be conducted in a target host animal to determine efficacy and long-term protection. Based on our initial data, we believe a gidA mutant STM strain used in a live-attenuated vaccine could provide superior protection against highly lethal levels of Salmonella by stimulating humoral, cellular immunity and potentially mucosal immunity.

\section{Conclusions}

Immunization with the gidA mutant STM strain provided full protection from a lethal dose challenge of WT STM. Sera levels of IgG2a and IgG1 were significantly higher in immunized mice when compared to sera of control mice, and the level of IgG1 showed a marked increase over IgG2a in the sera of immunized mice. Naïve mice receiving sera and cells from immunized mice were only partially protected from a lethal dose challenge of WT STM with the sera being more protective than the cells. A lymphocyte proliferation assay showed a marked response of splenocytes from immunized mice to treatment with STM cell lysate. Furthermore, the Th1 (IL-2 and IFN- $\gamma$ ) and Th2 (IL-10) cytokines showed a significant increase in the cell culture supernatant of splenocytes of immunized mice when treated with STM cell 
lysate. These data indicated the gidA mutant vaccine strain protects mice by inducing humoral and cellular immune responses with the humoral immune response being the primary mechanism of protection.

\section{Competing interests}

The authors disclose no conflicts of interest.

\section{Authors' contributions}

DS participated in the design of the study, carried out the experimental work, performed the statistical analysis, and drafted the manuscript. AF designed and coordinated the study, and edited the manuscript. All authors read and approved the final manuscript.

\section{Acknowledgements}

The authors would like to express their gratitude to Dr. Gary Splitter's research group, University of Wisconsin-Madison, for their technical assistance. The help of Dr. James Will, University of Wisconsin-Madison, in reviewing the manuscript is greatly appreciated. This work was supported by a grant from USDA Hatch Fund \#WIS1380.

Received: 22 July 2012 Accepted: 26 November 2012

Published: 30 November 2012

\section{References}

1. Scallan E, Hoekstra RM, Angulo FJ, Tauxe RV, Widdowson MA, Roy SL, Jones $J$, Griffin PM: Foodborne illness acquired in the United States-major pathogens. Emerg Infect Dis 2011, 17(1):7-15.

2. Becker D, Selbach M, Rollenhagen C, Ballmaier M, Meyer TF, Mann M, Bumann D: Robust Salmonella metabolism limits possibilities for new antimicrobials. Nature 2006, 440(7082):303-307.

3. Gordon MA, Graham SM, Walsh AL, Wilson L, Phiri A, Molyneux E, Zijlstra EE, Heyderman RS, Hart CA, Molyneux ME: Epidemics of invasive Salmonella enterica serovar enteritidis and S. enterica Serovar typhimurium infection associated with multidrug resistance among adults and children in Malawi. Clin Infect Dis 2008, 46(7):963-969.

4. Kwon YM, Cox MM, Calhoun LN: Salmonella-based vaccines for infectious diseases. Expert Rev Vaccines 2007, 6(2):147-152.

5. Kantele A, Arvilommi H, Kantele JM, Rintala L, Makela PH: Comparison of the human immune response to live oral, killed oral or killed parenteral Salmonella typhi TY21A vaccines. Microb Pathog 1991, 10(2):117-126.

6. Muotiala A, Hovi M, Makela PH: Protective immunity in mouse salmonellosis: comparison of smooth and rough live and killed vaccines. Microb Pathog 1989, 6(1):51-60

7. Mastroeni P, Chabalgoity JA, Dunstan SJ, Maskell DJ, Dougan G: Salmonella: immune responses and vaccines. Vet J 2001, 161(2):132-164.

8. Raupach B, Kaufmann SH: Bacterial virulence, proinflammatory cytokines and host immunity: how to choose the appropriate Salmonella vaccine strain? Microbes Infect 2001, 3(14-15):1261-1269.

9. Dunstan SJ, Simmons CP, Strugnell RA: Comparison of the abilities of different attenuated Salmonella typhimurium strains to elicit humoral immune responses against a heterologous antigen. Infect Immun 1998, $66(2): 732-740$

10. Liu T, Konig R, Sha J, Agar SL, Tseng CT, Klimpel GR, Chopra AK: Immunological responses against Salmonella enterica serovar Typhimurium Braun lipoprotein and lipid A mutant strains in SwissWebster mice: potential use as live-attenuated vaccines. Microb Pathog 2008, 44(3):224-237.

11. Matsuda $K$, Chaudhari AA, Lee JH: Evaluation of safety and protection efficacy on cpxR and lon deleted mutant of Salmonella Gallinarum as a live vaccine candidate for fowl typhoid. Vaccine 2011, 29(4):668-674.

12. Shippy DC, Eakley NM, Bochsler PN, Chopra AK, Fadl AA: Biological and virulence characteristics of Salmonella enterica serovar Typhimurium following deletion of glucose-inhibited division (gidA) gene. Microb Pathog 2011, 50(6):303-313.

13. von Meyenburg K, Jorgensen BB, Nielsen J, Hansen FG: Promoters of the atp operon coding for the membrane-bound ATP synthase of Escherichia coli mapped by Tn10 insertion mutations. Mol Gen Genet 1982, 188(2):240-248.
14. White DJ, Merod R, Thomasson B, Hartzell PL: GidA is an FAD-binding protein involved in development of Myxococcus xanthus. Mol Microbiol 2001, 42(2):503-517.

15. Elseviers D, Petrullo LA, Gallagher PJ: Novel Ecoli mutants deficient in biosynthesis of 5-methylaminomethyl-2-thiouridine. Nucleic Acids Res 1984, 12(8):3521-3534.

16. Bregeon D, Colot V, Radman M, Taddei F: Translational misreading: a tRNA modification counteracts a +2 ribosomal frameshift. Genes Dev 2001, 15(17):2295-2306.

17. Meyer S, Wittinghofer A, Versees W: G-domain dimerization orchestrates the tRNA wobble modification reaction in the MnmE/GidA complex. J Mol Biol 2009, 392(4):910-922.

18. Yim L, Moukadiri I, Bjork GR, Armengod ME: Further insights into the tRNA modification process controlled by proteins $\mathrm{MnmE}$ and GidA of Escherichia coli. Nucleic Acids Res 2006, 34(20):5892-5905.

19. Moukadiri I, Prado S, Piera J, Velazquez-Campoy A, Bjork GR, Armengod ME: Evolutionarily conserved proteins MnmE and GidA catalyze the formation of two methyluridine derivatives at tRNA wobble positions. Nucleic Acids Res 2009, 37(21):7177-7193.

20. Sha J, Kozlova EV, Fadl AA, Olano JP, Houston CW, Peterson JW, Chopra AK: Molecular characterization of a glucose-inhibited division gene, gidA, that regulates cytotoxic enterotoxin of Aeromonas hydrophila. Infect Immun 2004, 72(2):1084-1095.

21. Cho KH, Caparon MG: tRNA modification by GidA/MnmE is necessary for Streptococcus pyogenes virulence: a new strategy to make live attenuated strains. Infect Immun 2008, 76(7):3176-3186.

22. Gupta R, Gobble TR, Schuster M: GidA posttranscriptionally regulates rhl quorum sensing in Pseudomonas aeruginosa. J Bacteriol 2009, 191(18):5785-5792.

23. Kinscherf TG, Willis DK: Global regulation by gidA in Pseudomonas syringae. J Bacteriol 2002, 184(8):2281-2286.

24. Shippy DC, Heintz JA, Albrecht RM, Eakley NM, Chopra AK, Fadl AA: Deletion of glucose-inhibited division (gidA) gene alters the morphological and replication characteristics of Salmonella enterica Serovar typhimurium. Arch Microbiol 2012, 194(6):405-412.

25. Padhye NV, Doyle MP: Production and characterization of a monoclonal antibody specific for enterohemorrhagic Escherichia coli of serotypes 0157:H7 and O26:H11. J Clin Microbiol 1991, 29(1):99-103.

26. Niles AL, Moravec RA, Eric Hesselberth P, Scurria MA, Daily WJ, Riss TL: A homogeneous assay to measure live and dead cells in the same sample by detecting different protease markers. Anal Biochem 2007, 366(2):197-206.

27. John B, Harris TH, Tait ED, Wilson EH, Gregg B, Ng LG, Mrass P, Roos DS, Dzierszinski F, Weninger W, et al: Dynamic Imaging of CD8(+) T cells and dendritic cells during infection with Toxoplasma gondii. PLOS Pathog 2009, 5(7):e1000505.

28. Li Y, Wang S, Scarpellini G, Gunn B, Xin W, Wanda SY, Roland KL, Curtiss R 3rd: Evaluation of new generation Salmonella enterica serovar Typhimurium vaccines with regulated delayed attenuation to induce immune responses against PspA. Proc Natl Acad Sci USA 2009, 106(2):593-598.

29. Sprent J: Immunological memory. Curr Opin Immunol 1997, 9(3):371-379.

30. Shi R, Villarroya M, Ruiz-Partida R, Li Y, Proteau A, Prado S, Moukadiri I, Benitez-Paez A, Lomas R, Wagner J, et al: Structure-function analysis of Escherichia coli MnmG (GidA), a highly conserved tRNA-modifying enzyme. J Bacteriol 2009, 191(24):7614-7619.

31. Bohme S, Meyer S, Kruger A, Steinhoff HJ, Wittinghofer A, Klare JP: Stabilization of $\mathrm{G}$ domain conformations in the tRNA-modifying MnmE-GidA complex observed with double electron electron resonance spectroscopy. J Biol Chem 2010, 285(22):16991-17000.

32. Persson BC: Modification of tRNA as a regulatory device. Mol Microbiol 1993, 8(6):1011-1016.

33. Nielsen H, Engelbrecht J, Brunak S, von Heijne G: Identification of prokaryotic and eukaryotic signal peptides and prediction of their cleavage sites. Protein Eng 1997, 10(1):1-6.

34. Mastroeni P, Simmons C, Fowler R, Hormaeche CE, Dougan G: Igh-6(-/-) (B-cell-deficient) mice fail to mount solid acquired resistance to oral challenge with virulent Salmonella enterica serovar typhimurium and show impaired Th1 T-cell responses to Salmonella antigens. Infect Immun 2000, 68(1):46-53. 
35. McSorley SJ, Jenkins MK: Antibody is required for protection against virulent but not attenuated Salmonella enterica serovar typhimurium. Infect Immun 2000, 68(6):3344-3348.

36. Mittrucker HW, Raupach B, Kohler A, Kaufmann SH: Cutting edge: role of B lymphocytes in protective immunity against Salmonella typhimurium infection. J Immunol 2000, 164(4):1648-1652.

37. Carsetti R, Rosado MM, Wardmann H: Peripheral development of B cells in mouse and man. Immunol Rev 2004, 197:179-191.

38. Sad S, Mosmann TR: Single IL-2-secreting precursor CD4 T cell can develop into either Th1 or Th2 cytokine secretion phenotype. I Immunol 1994, 153(8):3514-3522.

39. Swain SL, Weinberg AD, English M, Huston G: IL-4 directs the development of Th2-like helper effectors. J Immunol 1990, 145(11):3796-3806.

40. Okahashi N, Yamamoto M, Vancott JL, Chatfield SN, Roberts M, Bluethmann $\mathrm{H}$, Hiroi T, Kiyono H, McGhee JR: Oral immunization of interleukin-4 (IL-4) knockout mice with a recombinant Salmonella strain or cholera toxin reveals that CD4+ Th2 cells producing IL- 6 and IL-10 are associated with mucosal immunoglobulin A responses. Infect Immun 1996, 64(5):1516-1525.

41. Hess J, Ladel C, Miko D, Kaufmann SH: Salmonella typhimurium aroAinfection in gene-targeted immunodeficient mice: major role of CD4+ TCR-alpha beta cells and IFN-gamma in bacterial clearance independent of intracellular location. J Immunol 1996, 156(9):3321-3326.

42. McSorley SJ, Cookson BT, Jenkins MK: Characterization of CD4+ T cell responses during natural infection with Salmonella typhimurium. J Immunol 2000, 164(2):986-993.

43. Mastroeni P, Villarreal-Ramos B, Hormaeche CE: Role of T cells, TNF alpha and IFN gamma in recall of immunity to oral challenge with virulent salmonellae in mice vaccinated with live attenuated aro- Salmonella vaccines. Microb Pathog 1992, 13(6):477-491.

44. Nauciel C: Role of CD4+ T cells and T-independent mechanisms in acquired resistance to Salmonella typhimurium infection. J Immunol 1990, 145(4):1265-1269.

45. Mizuno $Y$, Takada H, Nomura A, Jin CH, Hattori H, Ihara K, Aoki T, Eguchi $\mathrm{K}$, Hara T: Th1 and Th1-inducing cytokines in Salmonella infection. Clin Exp Immunol 2003, 131(1):111-117.

46. Ugrinovic S, Menager N, Goh N, Mastroeni P: Characterization and development of T-Cell immune responses in B-cell-deficient (Igh-6(-/-)) mice with Salmonella enterica serovar Typhimurium infection. Infect Immun 2003, 71(12):6808-6819.

doi:10.1186/1471-2180-12-286

Cite this article as: Shippy and Fadl: Immunological characterization of a gidA mutant strain of Salmonella for potential use in a live-attenuated vaccine. BMC Microbiology 2012 12:286.

\section{Submit your next manuscript to BioMed Central and take full advantage of:}

- Convenient online submission

- Thorough peer review

- No space constraints or color figure charges

- Immediate publication on acceptance

- Inclusion in PubMed, CAS, Scopus and Google Scholar

- Research which is freely available for redistribution 